Bol. Soc. Bot. México 49:7i-17 (1989)

\title{
Nutrición mineral
}

\author{
EMmanUel Rincon y PILAR Huante ${ }^{1}$
}

\begin{abstract}
RESUMEN. Estudios a nivel de la interfase raíz/suelo, distribución espacio-temporal de nutrientes y captura y uso de recursos, incluyendo estudios de forrageo, son sugeridos en un intento por motivar investigaciones experimentales, con un enfoque ecofisiológico, dentro del área de nutrición mineral en plantas.
\end{abstract}

ABSTRACT. Studies of the interfase root/soil, spatial-temporal distribution of nutrients and use and capture of resources, including foraging investigations are suggested in an attempt to encourage experimental research, with an ecophysiological approach, in the area of plant mineral nutrition.

\section{INTRODUCCION}

Los estudios ecofisiológicos en nutrición mineral buscan, en su mayoría, interpretar los patrones de distribución de las plantas relacionando las características nutricionales del ambiente y los atributos o mecanismos de las especies que les pemiten sobrevivir y reproducirse en ese ambiente en particular. Para lograr este objetivo, diferentes enfoques y métodos han sido adoptados dentro de las diferentes áreas de nutrición mineral, incluyendo estudios descriptivos de campo y laboratorio. Diferentes niveles de organización biológica han sido igualmente incluidos, abarcando desde actividad enzimática a nivel celular hasta ciclaje de nutrientes en ecosistemas. Cualquiera que sea el enfoque o disciplina desde la cual se aborde el problema de nutrición mineral, el investigador debe reconocer que el ambiente nutricional donde crece una planta es un factor importante que afecta la distribución y abundancia de las especies en la comunidad (Bradshaw, 1969).

1 Centro de Ecología, Universidad Nacional Autónoma de México, Apdo. Postal 70-275, Delegación Coyoacán 04510, México, D. F. 
En este artículo pretendemos realizar una revisión del área de nutrición mineral en plantas desde un punto de vista ecofisiológico, contrastando con el enfoque tradicional agronómico, el cual centra su interés en obtener gran cantidad y cierta calidad de algunas partes de la planta y no en la sobrevivencia y continuidad de una especie dentro de una comunidad natural (Chapin, 1980; Vitousek, 1982).

Este trabajo discute algunos de los problemas relacionados con medir experimentalmente la respuesta de las plantas a variaciones espacio-temporales en el suministro de nutrimentos. Los objetivos de este trabajo no contemplan, sin embargo, realizar un detallado análisis del papel e importancia de cada uno de los elementos minerales que intervienen en el metabolismo y crecimiento de las plantas. Tampoco pretendemos incursionar en el área de nutrición por carbono y fijación de nitrógeno por microorganismos. Información de este tipo puede encontrarse en los trabajos de Brownell (1979); Clarkson y Hanson (1980), Marschner (1983), Lee, McNeill y Rorison (1983), Harley y Smith (1983); Moser y Haselwander (1983); Kirby y Pilbeam (1984); Clarkson (1985), y los artículos de la Dra. Valdés y el Dr. Castellanos en este volúmen.

\section{ENFOQUE EXPERIMENTAL}

Dentro de esta sección tratamos de explorar algunos de los problemas que surgen al estimar la respuesta de plántulas a cambios en su ambiente nutricional cuando éstas son cultivadas en invernaderos o cuartos de crecimiento. No obstante el exceptisismo de algunos ecólogos (Harper, 1961) acerca de la utilidad de la información obtenida mediante experimentos de laboratorio, dentro del área de nutrición mineral el enfoque experimental ha demostrado ser muy valioso, aportando resultados comparativos de alto valor predictivo (Bradshaw et al., 1964; Grime y Hodgson, 1969; Austin y Austin, 1980). Sin embargo, es necesario considerar, antes de iniciar cualquier estudio de laboratorio, algunos aspectos metodológicos que puedan afectar el resultado de los experimentos, así como tener siempre presente que los estudios de campo, principalmente aquellos de caracter edáfico, deben preceder a cualquier tipo de estudio experimental. Estos aspectos metodológicos son: las técnicas y métodos experimentales adoptados dentro de una investigación, la fase del ciclo de vida a estudiar en una especie y la selección de las variables of factores a estimar.

Uno de los avances técnicos mas importantes en el estudio de nutrición mineral es el surgimiento de los denominados cuartos o cámaras de crecimiento (Clapham, 1956; Evans, 1963; Grime y Hunt, 1975). En la actualidad es posible realizar series de experimentos bajo condiciones controladas y facilmente repetibles. El uso de cuartos de crecimiento en nutrición mineral tiene dos ventajas sobresalientes; los efectos de diferentes tipos y concentraciones de nutrimentos pueden ser facilmente probados, vía cultivos en solución u otros sustratos (Hewitt, 1966; Adams, 1974; Asher y Edwards, 1983) y la interacción entre factores climáticos y nutricionales puede ser estimada con mayor precisión. El uso de cuartos de crecimiento facilita también simulaciones experimentales como sería el caso de estudios de competencia o bien captura de recursos en ambientes heterogéneos (Grime et al., 1986). 
Fase del ciclo de vida.

La etapa del ciclo de vida que comprende germinación y establecimiento se considera de alto riesgo (Rorison, 1969; Harper, 1977) para la sobreviviencia y permanencia de una especie dentro de una comunidad. Es en esta etapa donde la respuesta de la especie a diferentes tratamientos se espera que sea más sensible y también cuando la influencia de factores ambientales puede ser más facilmente detectable. Con cualquier especie dos factores deben ser considerados al realizar experimentos a nivel de plántula. Estos son requerimientos de germinación y tamaño y reservas de la semilla. Es necesario conocer los requerimientos de germinación de la especie para poder obtener al mismo tiempo el suficiente número de plántulas con características similares (edad, número de hojas, tamaño). El tamaño de la semilla y sus reservas influirán directamente en el vigor de la plántula y el tiempo de respuesta de la misma al tratamiento.

Alternativamente, los experimentos se pueden realizar con "clones", si la especie estudiada se reproduce principalmente en forma vegetativa. Especial cuidado se debe dar en este caso a la colecta de la especie y su estatus nutricional, ya que estos factores pueden alterar los resultados de cualquier tipo de experimentos, más aún cuando se consideran nutrimentos como nitrógeno y fósforo los cuales son móviles dentro de la planta (Clarkson, 1967; Runge, 1983; Robinson y Rorison, 1983b; Lee et al., 1983). Pretratamiento de los clones debe verse también con cuidado, ya que durante este proceso se puede estar induciendo un estado de tole-ancia a altos niveles de nutrimentos y resistencia (déficit) de los individuos, que sin duda influirá en posteriores resultados.

\section{Factores a evaluar.}

Los estudios de nutrición mineral de carácter ecofisiológico pretenden identificar posibles adaptaciones de las especies a condiciones edáficas extremas (Chapin, 1980; Chapin, Vitousek y Van Cleve, 1986). Estas investigaciones buscan evaluar las respuestas de las especies a condiciones pobres y heterogéneas en el suministro de nutrimentos (Robinson y Rorison, 1983a; Grime et al., 1986; Rorison, 1987). Estas respuestas pueden ser evaluadas en términos de crecimiento y captura de nutrimentos. En lo que se refiere a evaluar la respuesta de las plantas a diferentes tratamientos especial cuidado debe darse al diseño del experimento (Chrochan y Cox, 1957) y análisis de los resultados. La figura 1 muestra cómo un peso seco "X" puede ser alcanzado en un tiempo "Y" (Evans, 1972; Hunt, 1982; Causton y Venus, 1981) con tres diferentes velocidades. Este ejemplo realza la importancia de estimar la respuesta de las plantas mediante más de una cosecha siguiendo el enfoque funcional en análisis de crecimiento. Estimaciones de la tasa relativa de crecimiento (Grime y Hunt, 1975) en plántulas nos permitirá evaluar la capacidad o velocidad a la que una plántula captura y transforma energía y materiales. Dependiendo para su establecimiento, pemanencia y reemplazamiento dentro de la comunidad, de su habilidad para responder a cambios en el ambiente y la asignación de sus recursos (crecimiento "vs" reproducción) (Abrahams, 1982). Fig. 1.

La captura de nutrimentos estimada mediante análisis químico de raíces y tallos en forma independiente (Allen, Grinshaw y Rowlan, 1986) nos proporciona dos tipos de 
información; a) qué y cuánto un individuo ha absorbido o capturado de su ambiente nutricional en un tiempo determinado y b) cuánto de eso que ha capturado ha sido transferido de la raíz a la parte aérea. Es importante estudiar tanto la raíz como la parte aérea de la planta, para lograr obtener la información que nos permita evaluar el efecto diferencial de disponibilidad de nutrimentos y su asignación tanto a raíces como a hojas $\mathrm{y}$ tallos.

\section{RAICES}

Con la evolución de las plantas superiores a partir de un medio acuático a uno terrestre, las raíces adquirieron un doble papel: anclaje y soporte de la planta al sustrato e intercambio de materiales con el suelo (Pitman 1976; Epstein, 1972, 1976). La estructura y distribución de las raíces en tiempo y espacio debe cumplir con estas dos funciones (Luttge, 1983). La Figura 2 muestra la compleja estructura de la raíz, la cual se espera que optimece las funciones de captura y transporte de agua y minerales, además de desempeñar funciones de anclaje (Grime et al., 1986). A pesar de la gran importancia de los estudios estructurales y funcionales en raíces (Fitter, 1985), poca atención ha recibido el estudio de raíces a nivel ecológico, posiblemente por los grandes problemas técnicos y metodológicos que se presentan (Bohm, 1979). En estudios de nutrición mineral en plantas no es sorprendente que las raíces reciban mayor atención por ser éstas los órganos de absorción de mayor importancia en plantas vasculares. Tradicionalmente, estudios de captura de nutrientes se han concentrado en investigaciones de los mecanismos de incorporación de iones, como serían estudios sobre mecanismos de acarreo, o bien, sitios de unión múltiple y selectividad iónica (Epstein y Hagen, 1952; Epstein, 1976; Brownell, 1978; Clarkson, 1985). Mayor atención, sin embargo se está dando a estudios electrofisiológicos en un intento por explicar aspectos adaptativos en ambientes donde tolerancia a déficit nutricional es importante o bien optimización del proceso de captura de nutrimentos afecta la interacción entre especies. De esta manera nuevos campos de investigación en ecofisiología de raíces están surgiendo para explicar patrones de distribución de plantas silvestres. Un ejemplo de estos enfoques lo constituyen las investigaciones realizadas sobre el efecto de $\mathrm{pH}$ (Rorison, 1987), fuente de nitrógeno (Runge, 1983; Robinson y Rorison, 1983a, b) y captura de fósforo. También diferencias en captura de aniones y cationes y el consecuente cambio en $\mathrm{pH}$ alrededor de la raíz y su efecto en captura de fósforo están siendo activamente investigados. Conjuntamente y a pesar de los problemas metodológicos involucrados (Bohm, 1979) se está investigando más acerca del crecimiento de raíces en espacio y tiempo, ya que este proceso es de gran importancia para la nutrición de las plantas (Troughton, 1983). Además de reconocer la importancia de la interfase raíz-suelo y la concentración de iones en el sustrato, particular atención se está dando a las tasas de difusión de ciertos minerales, como potasio (Hawker et al. 1974) y fósforo ya que es su tasa de difusión en el sustrato lo que limita su incorporación al metabolísmo de la planta y no particularmente su disponibilidad total en el medio. Aún más recientes son los estudios de plasticidad y forrageo de raíces que se están realizando con el fin de relacionar aspectos de costo-beneficio al capturar recursos en diferentes ambientes y estimar la capacidad 


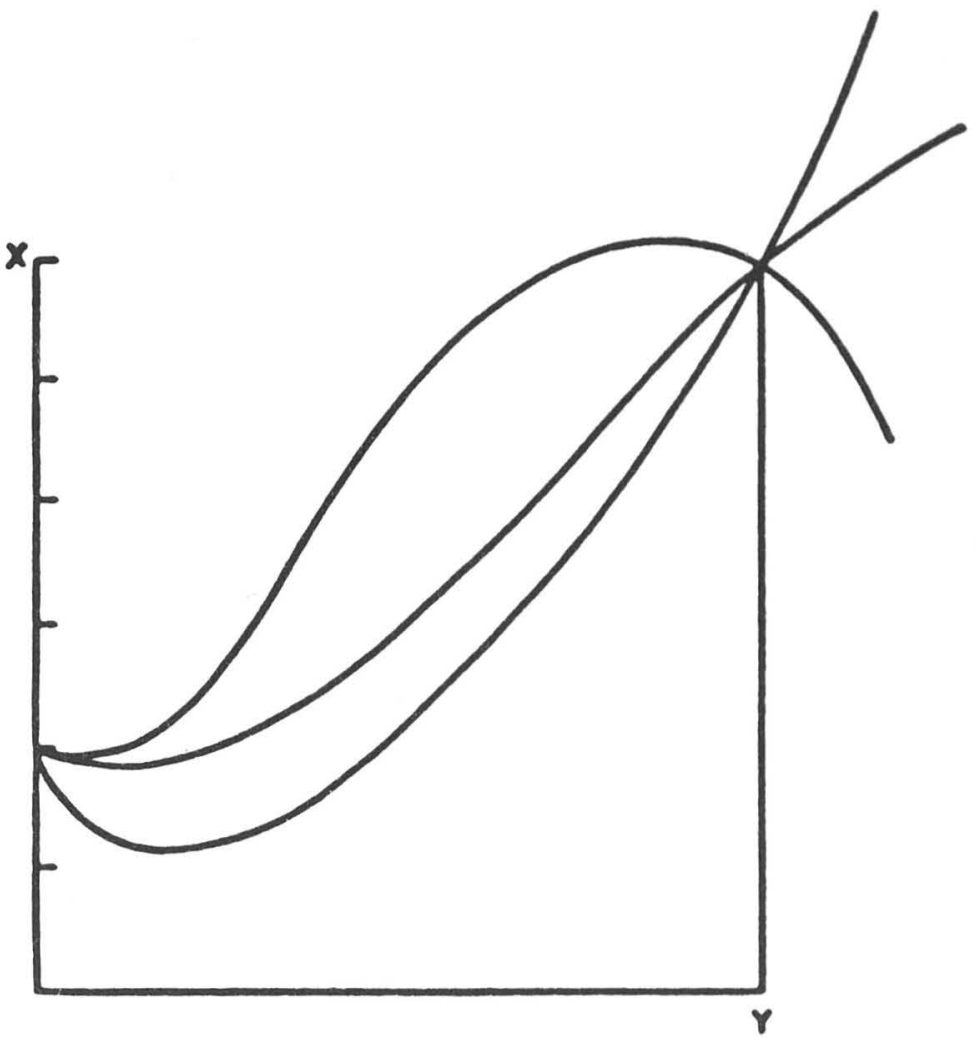

Figura 1. Representación de tres diferentes curvas de crecimiento las cuales alcanzan un mismo valor de $x$ a un tiempo $y$. 


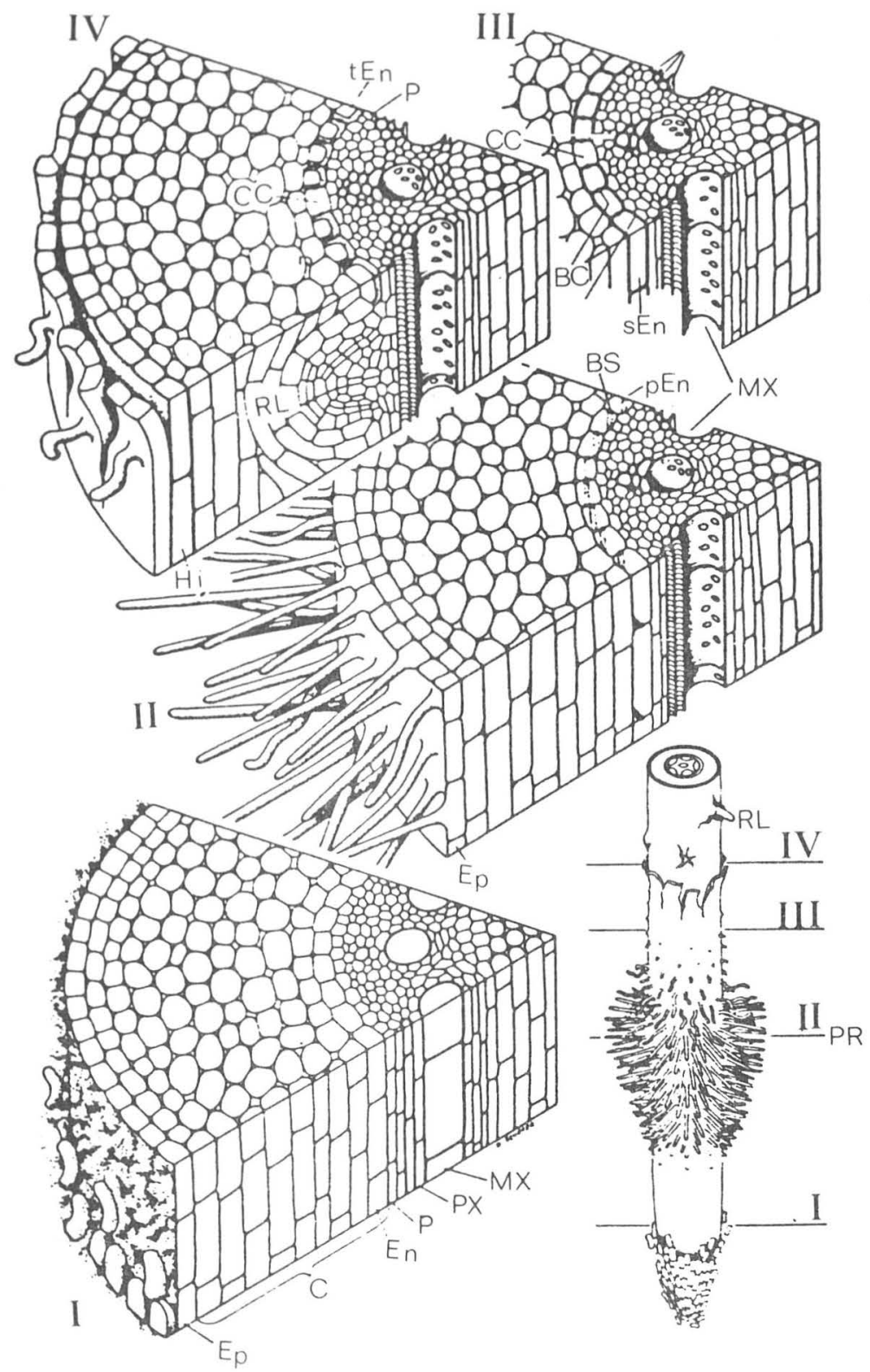

Figura 2. I-IV Esquemas tridimensionales de una raíz con detalles de cuatro zonas radiculares sucesivas. La anatomía de una raíz de pasto fue tomada como base para el esquema; las distancias a lo largo de la raíz no son indicadas porque la diferenciación puede ser muy variable, dependiendo de la especie y las condiciones de crecimiento. 1) Zona de diferenciación primaria, II) Zona de pelos radiculares con endodermis primaria, III) Zona de endodermis secundaria, IV) Zona de formación lateral de la raíz, hipodermis y endodermis terciaria. C corteza, BC bandas de Caspari, En endodermis (pEn primaria, sEn secundaria, tEn terciaria); Ep epidermis, Hi hipodermis, RL raíz lateral, MX metaxilema, $\mathrm{P}$ periciclo, cc conducto celular, F floema, PX protoxilema, PR pelos radicales, LS lamela suberizada, PX parénquima xilemático. 
diferencial de las especies para responder a cambios en su ambiente nutricional y su ambiente aéreo, en particular cambios espacio-temporales en luz (Bloom et al., 1985; Grime et al., 1986; Slade y Hutchings, 1987). Fig. 2.

Estudios comparativos de laboratorio en los que la respuesta de diferentes especies a un mismo ambiente nutricional pueden ser evaluadas nos ayudará a identificar preguntas que nos auxilien en el diseño de experimentos tendientes a entender los diferentes mecanismos de las especies en la captura y uso de recursos minerales. Entre estos estudios debemos incluir investigaciones de crecimiento de raíces abarcando su morfología y arquitectura (Troughton 1983; Fitter 1985).

\section{ELEMENTOS MINERALES}

Aquellos elementos minerales que son necesarios para lograr crecimiento y reproducción, que no pueden ser reemplazados por otro elemento y que tienen un efecto directo sobre el metabolismo de las plantas son considerados esenciales. En la tabla 1 se listan los elementos minerales metabólicamente esenciales junto con algunas de sus funciones. De los 16 elementos esenciales (C, O, H, N, P, Ca, S, Mg, Mn, Mo, Fe, B, Cu, $\mathrm{K}, \mathrm{Zn}, \mathrm{Cl}$ ) los dos primeros provienen de la atmósfera, los restantes son adquiridos en su mayoría a partir del sustrato. Existen también otros elementos que las plantas pueden absorber del sustrato como $\mathrm{Pb}, \mathrm{Cd}, \mathrm{Al}, \mathrm{Ni}, \mathrm{Se}, \mathrm{Cr}, \mathrm{F}, \mathrm{Na}$, y Co. Estos elementos sin embargo, no son estrictamente esenciales y en algunos casos pueden ser tóxicos a bajas concentraciones. Los elementos que estimulan crecimiento sin ser esenciales o que son esenciales sólo para ciertas familias o especies son considerados elementos benéficos. Dentro de este grupo encontramos al Sodio, Silice y Cobalto (Yoshida et al., 1969; Yuan y Chang, 1978; Brownell, 1978). El sodio que es considerado como micronutriente para plantas C4 (Brownell, 1979) es de particular interés ya que tiene un efecto benéfico en plantas que crecen en lugares donde el Potasio es pobre (Marshener y Possingham, 1975) y es nocivo para plantas como Phaseolus vulgaris (Hawker et al., 1974). Este tipo de efecto contrastante hace necesario obtener mas información sobre el papel del sodio en nutrición mineral. Más aún si se considera que el sodio es abundante en la mayoría de los sistemas naturales y en el agua de irrigación de las regiones áridas (Epstein et al., 1980). Tabla 1.

Los estudios a nivel biofísico y bioquímico que se han realizado con diferentes iones han contribuido a explicar las diferentes vías de captura de iones inorgánicos, así como también han incrementado nuestro entendimiento acerca de los procesos energéticos que intervienen en el transporte a través de la membrana celular y el papel de los elementos minerales en la regulación de procesos metabólicos. Muchos de estos estudios se han realizado gracias a costosas técnicas con radioisótopos y la incorporación de conceptos de cinética enzimática (Epstein y Hagen, 1952; Epstein, 1972). De esta manera se ha podido establecer algunas de las funciones de diferentes elementos minerales, como es la función de Fe como agente acarreador de electrones o bien induciendo cambios en la configuración molecular de enzimas o su sustrato como es el caso de $\mathrm{Mg}$ y $\mathrm{K}$ (Marschner, 1983). No obstante lo importante de estos avances, es necesario considerar que la mayoría de estos estudios están basados en experimentos in 
vitro, lo cual limita nuestras interpretaciones acerca del papel de ciertos elementos minerales en la regulación de los cambios morfológicos y fisiológicos a nivel del individuo creciendo en su ambiente natural.

Tabla 1. Algunas funciones de nutrimentos minerales en plantas vasculares.

\begin{tabular}{|c|c|c|c|}
\hline Elemento & $\mathrm{mol} / \mathrm{g}^{\mathrm{a}}$ & $\begin{array}{l}\text { Constituyente de } \\
\text { compuestos orgáni- } \\
\text { cos esenciales }\end{array}$ & $\begin{array}{l}\text { Activador }(+) \text { o } \\
\text { inhibidor }(-) \text { de } \\
\text { enzimas o reacciones } \\
\text { enzimática }\end{array}$ \\
\hline $\mathrm{N}$ & 1000 & $\begin{array}{l}\text { Proteínas, ácidos } \\
\text { nucléicos }\end{array}$ & $\begin{array}{l}\text { Nitrato reductasa } \\
\mathrm{NO}_{3}(+), \mathrm{NH}_{4}(-)\end{array}$ \\
\hline S & 30 & $\begin{array}{l}\text { Proteínas, } \\
\text { Sulfolípidos }\end{array}$ & \\
\hline$P$ & 60 & $\begin{array}{l}\text { Acidos nucléicos, } \\
\text { coenzimas }\end{array}$ & $\begin{array}{l}\text { ADPG-pirofosforilasa } \\
(-) \\
\text { Amilofosforilasa }\end{array}$ \\
\hline $\mathrm{K}$ & 250 & Desconocido & Amilo sintetasa \\
\hline $\mathrm{Ca}$ & 125 & Pectatos & Amilasa (+) \\
\hline $\mathrm{Mg}$ & 80 & Clorofila & $\begin{array}{l}\text { Fosforilación }(+) \\
\text { RubP-carboxilasa }\end{array}$ \\
\hline $\mathrm{Fe}$ & 2 & $\begin{array}{l}\text { Ferredoxina, } \\
\text { citocromo }\end{array}$ & \\
\hline $\mathrm{Mn}$ & 1 & Desconocido & $\begin{array}{l}\text { Reacción de Hill }(+) \\
\text { Aminopeptidasas }(+)\end{array}$ \\
\hline $\mathrm{Zn}$ & 0.3 & $\begin{array}{l}\text { Anhidrasa carbonica } \\
\text { Fosfotasa alcalina }\end{array}$ & $\begin{array}{l}\text { Hexoquinasa }(+) \\
\text { Ribonucleasa (-) }\end{array}$ \\
\hline $\mathrm{Cu}$ & 0.1 & $\begin{array}{l}\text { Acido ascórbico } \\
\text { oxidasa, } \\
\text { Plastocianina }\end{array}$ & \\
\hline B & 2 & Desconocido & \\
\hline $\mathrm{Cl}$ & 13 & Desconocido & \\
\hline Mo & 0.001 & Nitrato reductasa & \\
\hline
\end{tabular}

a

Concentración promedio de materia seca suficiente para un crecimiento adecuado. 
A pesar de la gran importancia de los estudios de nutrición mineral en agricultura, es sorprendente que en México esta área esté tan poco explorada (Larque, 1987). La gran diversidad de climas, compleja geografía y diversidad de comunidades vegetales (Rzedowski, 1978) presentan un gran número de problemas de índole nutricional, entre ellos podemos citar los estudios descriptivos a nivel edáfico, o bien disponibilidad de recursos en tiempo y espacio, estudios de tolerancia a déficit de nutrientes, estudios combinados con forrageo y optimización de uso de recursos, estudios de raíces, utilización de nitrógeno en forma orgánica o inorgánica en ambientes lumínicos contrastantes y, aspectos de costo-beneficio en captura de nutrientes (Bloom, et al. 1985), sin mencionar los estudios a nivel agronómico, en los que se puede determinar el potencial fisiológico y ecológico de especies silvestres en proceso de domesticación.

\section{LITERATURA CITADA}

ABRAHAMS, W. G. 1982. On the comparative allocation of biomass, energy and nutrients in plants. Ecol. 63(4): 982-991.

ADAMS, F. 1974. Soil solution pp. 441-481. En: Carson E. W. (Edr) The plant root and its environment. University Press of Virginia, Charlottes Ville.

ALLEN, S. E., H. M. GRIMSHAW, y A. P. ROWLAND. 1986. Chemical analysis pp. 285-342. En: Moore, P. D. Methods in plant ecology. Chapman, London.

ASHER, C. J. y D. G. EDWARDS. 1983. Modern solution culture techniques. pp. 94-119. En: Lauchli A. y R. L. Bieleski (Edrs.). Inorganic plant nutrition. Encyclopedia of plant physiology. New series, Vol. 15 A. Springer-Verlag, Berlin.

AUSTIN, M. P. y B. D. AUSTIN. 1980. Behaviour of experimental plant communities along a nutrient gradient. J. Ecol. 68: 891-918.

BLOOM, A. J., F. S. CHAPIN, y H. A. MOONEY, 1985. Resource limitation in plants - an economic analogy. Ann. Rev. Ecol. Syst. 16: 363-392.

BOHM, W. 1979. Methods of Studying root systems. Ecological Studies 33. Springer-Verlag, Berlin.

BRADSHAW, A. D. 1969. An ecologist's view point. pp. 415-427 En: I. H. Rorison (Ed.) Ecological Aspects of the Mineral Nutrition of Plants. Simposia of the British Ecological Society, No. 9 Blackwell Scientific Publications, Oxford.

BRADSHAW, A. D., M. J. CHADWICK, D. JOWETT, y R. W. SNAYDON, 1964. Experimental investigations into the mineral nutrition of several grass species. IV. Nitrogen level. J. Ecol. 52: 665-676.

BROWNELL, P. F. 1978. Mechanisms of ion uptake by plants. Plant Cell and Environment 1: 249-257.

BROWNELL, P. F. 1979. Sodium as an essential micronutriment element for plants and its possible role in metabolism. Adv. Bot. Rev. 7: 117-224.

CAUSTON, D. R. y J. C. VENUS. 1981. The biometry of plant growth. Edward Arnold, London.

CHAPIN, F. S. 1980. The mineral nutrition of wild plants. Ann. Rev. Ecol. Syst. 11: 233-260.

CHAPIN, F. S., P. M. VITOUSEK, y K. VAN CLEVE. 1986. The nature of nutrient limitation in plant communities. Amer. Natur. 127 (1): 48-57. 
CLAPHAM, A. R. 1956. Autoecological studies and the biological flora of the British isles. J. Ecol. 44: $1-11$.

CLARKSON, D. T. 1967. Phosphorous supply and growth rate in species of Agrostis L. J. Ecol. 55: 111-118. CLARKSON, D. T. 1985. Factors affecting mineral nutrient acquisition by plants. Ann. Rev. Plant Physiol. 36: 77-115.

CLARKSON, D. T. y J. B. HANSO, 1980. The mineral nutrition of higher plants. Ann. Rev. Plant Physiol. 31: 239-298.

COCHRAN, W. G. y G. M. COX, 1957 Experimental Designs. John Wiley-Sons. New York. EPSTEIN, E. 1972. Mineral Nutrition of Plants: Principles and Perspectives. Wiley and Sons, New York. EPSTEIN, E. 1976. Kinetics of ion transport and the carrier concept. pp. $70-94$ En: Luttge, V. and Pitman, M. G. (Edrs.). Transport in plants 11, part B. Tissues and organs. Encyclopedia of plant physiology. New series Vol. II. Springer-Verlag, Berlin.

EPSTEIN, E. y C. E. HAGEN, 1952. A kinetic study of the absorption of alkali cations by barley roots. Plant Physiol. 27: 457-474.

EPSTEIN, E., J. D. NORLYN, E. W. RUSH, R. W. KINGSBURG, D. B. KELLEY, G. A. CUNNINGHAMS, y A. F. WRONA, 1980. Saline culture of crops. Gen. Appr. Sci. 210: 399-404

EVANS, L. T. 1963. Extrapolation from controlled environments to the field pp. 421-435. En: L. T. Evans (Edr). Environmental control of plant growth. Camberra.

EVANS, G. C. 1972. The quantitative analysis of plant growth. Blackwell Scientific Publications. Oxford. FTTTER, A. H. 1985. Functional significance of root morphology and root system architecture. pp. 87-106. En: Fitter, A. H.; Atkinson, D.; Read, R. J. y Usher, M. B. (Edrs.) Ecological Interactions in Soil Plants Microbes and Animals. British Ecological Society. Blackwell Scientific Publications.

GRIME, J. P. y R. HUNT, 1975. Relative growth rate: its range and adaptive significance in a local flora. J. Ecol. 63: 393-422.

GRIME, J. P. y J. G. HODGSON. 1969. An investigation of the ecological significance of lime-chlorosis by means of large-scale comparative experiments. p. 67. En: Rorison, I. H. (Edr) Ecological Aspects of the Mineral Nutrition of Plants. Blackwell Scientific Publications, Oxford.

GRIME, J. P., J. C. CRICK y J. E. RINCON. 1986. The Ecological Significance of Plasticity. pp. 5-29. En: Jennings, D. H. y Trewavas, A. J. (Edrs.) Plasticity in Plants. Symposia of the Society for Experimental Biology No. 40. University of Cambridge. U. K.

HARLEY, J. L. y S. E. SMITH. 1983. Mycorrhizal Symbiosis. Academic Press, London.

HARPER, J. L. 1961. Approaches to the study of plant competition. pp. 1-39. En: F. L. Milthorpe (Edr.). Mechanisms in Biological Competition. Symp. Soc. Exp. Biol., Cambridge University Press.

HARPER, J. L. 1977. The population biology of plants. Academic Press, London.

HAWKER, J. S., H. MARSCHNER y W. J. S. DOWNTON 1974. Effects of sodium and potasium on starch synthesis in leaves. Austr. J. Plant Physiol. 1: 491-501.

HEWITT, E. J. 1966. Sand and water culture methods used in the study of plant nutrition. Common Wealth Agricultural Bureaux. Technical Communication 22.

HUNT, R. 1982. Plant growth curves: The functional approach to plant growth analysis. Edward Arnold. London.

KIRBY, E. A. y D. J. PILBEAM, 1984. Calcium as a plant nutrient. Plant Cell and Environment 7: 397-405. LARQUE SAAVEDRA, A. 1987. Historia de la fisiología vegetal en México. Ciencia 38 (2): 109-118. 


\section{NUTRICION MINERAL}

LEE, J. A., S. MCNEILL y I. H. RORISON, 1983. Nitrogen as an Ecological Factor. 22nd. Symposium of the British Ecological Society. Blackwell Scientific Publications, Oxford.

LUTTGE, U. 1983. Import and export of mineral nutrients in plant roots. pp. 181-211. En: A. Lauchli y R. L. Bieleski (Edrs.) Inorganic plant nutrition. Encyclopedia of plant physiology 15 A. Springer-Verlag, Berlin.

MARSCHNER, H. 1983. General introduction to the mineral nutrition of plants. pp. 7-60. En: A. Lauchli y R. L. Bieleski (Edrs.) Inorganic plant nutrition. Encyclopedia of Plant Physiology. New Series Vol. 15 A. Springer Verlag, Berlin.

MARSCHNER, H. y J. V. POSSINGHAM. 1975. Effect of K and Na on growth of leaf discs of sugar bet and spinach. Z. Phlanzenphysiol. 85: 6-16.

MOSER, M. y K. HASELWANDTER. 1983. Ecophysiology of micorrhizal symbiosis, pp. 391-421. En: O. L. Lange, P. S. Nobel, C. B. Osmond y H. Ziegler (Edrs.) Physiological Plant Ecology III.Responses to the Chemical and Biological Environment. Encyclopedia of Plant Physiology. New Series Vol. 12 C. Springer Verlag, Berlin.

PITMAN, M. G. 1976. Ion uptake by plant roots. pp. 95-128. En: Luttge, V. y Pitman, M. G. (Edrs.). Transport in plants 11, part b. Tissues and organs. Encyclopedia of plant physiology. New series Vol. II. Springer, Berlin.

ROBINSON, D. y I. H. RORISON. 1983a. Relationships between root morphology and nitrogen availability in a recent theoretical model describing nitrogen uptake from soil. Plant Cell and Environment 6: 641-647.

ROBINSON, D. y I. H. RORISON. 1983b. A comparison of the responses of Lolium perenne L., Holcus lanatus L. and Deschampsia flexuosa (L.) Trin. to a localized supply of nitrogen. New Phytol 94 : 263-273.

RORISON, I. H. 1969. Ecological inferences from laboratory experiments on mineral nutrition. pp. 155-157. En: Rorison, I. H. (Edr.) Ecological Aspects of the Mineral Nutrition of Plants. Blackwell Scientific Publications, Oxford.

RORISON, I. H. 1987. Mineral nutrition in time and space. New Phytol. 106: 79-92.

RUNGE, M. 1983. Physiology and ecology of nitrogen nutrition. pp. 163-200. Encyclopedia of plant Physiology Vol. 12 C. Springer-Verlag, Berlin.

RZEDOWSKI, J. 1978. Vegetación de México. Ed. Limusa., México.

SLADE, A. J. y M. J. HUTCHINGS. 1987. The effect of nutrient availability on foraging in the clonal herb Glechoma hederacea. J. Ecol. 75: 95-112.

TROUGHTON, A. 1983. Root Ecology and its Practical Applicatic. pp. 447-452. Int. Symp. Gumpenstein. Bundesanstalt Gumpestein, A-8952 Irdning.

VITOUSEK, P. 1982. Nutrient cycling and nutrient use efficiency. Ameri. Natu. 119 (4): 553-572.

YOSHIDA, S., S. A. NASAVERO, y E. A. RAMIREZ; 1969. Effect of silica and nitrogen supply on some leaf characters of rice plant. Plant Soil 31: 48-56.

YUAN, H. F. y Y. S. CHANG. 1978. Effect of available silicon in paddy soil on the growth of rice plants. Bot. Bull. Acad. Sinica 19: 125-138. 